This report was prepared as an account of work sponsored by the United States Government. Neither the United Stutes nor the United Ststes tiomic Energy Commission, nor any of their employes. nor any of their contrac. tors, subcontrectors, or their employees, makes any warranty, express or im. plied, of asumes any legal liability or responsibility for the accurecy. com. pleteness of usefulness of any intomation, apparatus, product of process disclosed. or represents that its use would not infringe privately owned rights.

Printed in the United States of America. Avsibble from National Technical Information Service

U. S. Depariment of Commerce 5285 Port Royal Roed Springtield. Virginis $\$ 2151$

Price: Printed Copy S4.00 Nlicrofiche $\$ 1.45$ 


\section{A Unified Model of Solar System and Galaxy Formation Based on Explosive Fragmentation}

b?

Wilbur K. Brown

\begin{tabular}{|c|}
\hline 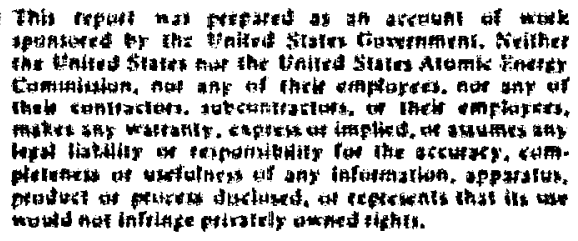 \\
\hline
\end{tabular}


A UNIFIED MODLL OF SOLAR SYSTEM AND GALAXY FORMATION

BASED ON EXPLOSIVE FRAGMENTATION

by

Wilbur K. Brown

\begin{abstract}
A new cogmogonicul model based on postulated fragmentation during postexplosion expansion is proposed. Application to both solat system and galaxy formation produces descriptions of systers closely resembing those presently observed.
\end{abstract}

\section{INTRODUCT ION}

A "good" model, by anyone's definition, is both simple and accurately descriptive of reality. It aay be fairly stated that no present model of galactic and solar system formation answers this description. (Noolfson gives an excellent revicw of proposed solar system-formation models; a recent article by oort ${ }^{2}$ includes the problems in models of galaxy formation.) The problems of constructing a cosmogonical model are manifold: Neither the formation process nor the event that initiated the process has been observed. The dynamics of the formation process are undoubtedly complex and appear amenable only to computer simulation. Tantalizingly, many galaxies may be obsezved, but with relatively Iittle detail; only one solar system is available for close scrutiny, and there we observe a bewildering azray of anomalies within a predominantly ordered system. A simple model that successfuliy describes both galactic and solar system formation has obvious appeal.

The present model is based on postulated fragmentation following an initiating explosive event: the big bang on the gaiactic scale and the supernova on the solar system scale. In both eases, it is assumed that prt-explosion compression brought the material to a viscous state of uniform density in which an organization was achieved. A patcern of motion, large in scale with respect to an individual fragment, can therefore be assigned to the material at the time of breakup. The model proposes that each fragment formed in the bis bang resulted in a galaxy, that cach massive fragment of an ejected supernova shell yiclds a complete solat system, and that on cither scale the final morphology is predestined by conditions existing at the moment of fragmentation. To avoid the conplexities of the dynamic analysis, the model has been constructed to bridge this phase and describe the end result directly: A fragment-to-disk trunsform is derived, based on the assumption that the angular momentun of cach particle is conserved in the transition--an assumption used earlier by Mestel. ${ }^{3}$ A gencral outline of the madel is given in a subsequent section. 
Recent, detailed articles haye separately described the application of the model on the solar system scale $e^{4}$ and the galactic scale. 5 The successful results of these efforts are reviewed in later sections of this article. Aside from this, the main purpose here is to emphasize the apparent universaity of the model which allows it to be critically compared not only to the available information on elliptical galaxies, but also to the wealth of observations concerning the solar system.

\section{AN ANALOGY}

Unification of the fragmentation

model on the solar system and galactic scales requires that a supernova and the big bang be considered analagous eyents. Because of conceptual difficulties involved in describing the big bang, it is easier to discuss the supernova and then make a logical extension to the big bang.

Picture a two-dimensional orjerve: 10cated in the finite but unbounded, rotating spherical surface of a presupernova star. As collapse begins, the observer has difficulty conceiving of the radial inward motion, but notes that the density of matter is increasing throughout his space. Subsequenty, a state of maximum density is reached, and the surface space is driven outward by energy from a source outside and at right angles to the observer's space, and yet enclosed by it. The surface density is now seen to decrease again; and, soon after, the surface separates into fragments. The fragments move apart from each other with a velocity proportional to the distance from the observer. The speed of expansion is slowed by the attraction of the star mass. The material within each fragment begins a swirling collapse toward the local center of mass as the space continues to expand. A complete disk-shaped system is formed from each fragment as they recede from each other.
To get an impression of the big bang, the reader is invited to read the foregoing paragraph while imagining a four-dimensional space rather than a three-dimensional one, and on the scale of the big bang.

III. THE FRAGMENTAT ION MODEL

Mathematical formulation of the fragmentation model is based on the set of postulates outlined in Table I. At the heart of the model is the assumption that at the time of fragmentation there is a bulk motion of the material--large in scale conpared to the size of an individual fragment--which arose from the pre-explosion compression. A linear velocity profile has been chosen to represent this large-scale motion locally. Figure 1 shows the derivation of the tangential velocity distribution, $v_{1}$, with respect to the fragment center of mass, starting with the 1 inear velocity profile in the bulk flow.

A cylindrical coordinate system, $r$, $\phi, 2$, is set up with the origin at a point that will become the center of mass of a fragment, with the plane defined $b y$ the $z$ and $r(\phi-0)$ axes oriented perpendicularly to the direction of the flow, and with the $r(\phi=0)$ axis along the direction of the profile gradient. The velocity profile is assimed to have no 2 -dependence, and is described by

$$
\left(\frac{\partial v_{1}}{\partial r_{1}}\right)_{\phi_{1}=0}=\frac{v_{0}}{r_{0}},
$$

where $v_{0}$ and $r_{0}$ are constants. The subscript 1 indicates conditions in the fragment at the time of separation. The extremely simple velocity distribution given by Eq. (1) describes a wide range of material motion from rigid body rotation to a good local approximation of shearing differential rotation. Inspection of Fig. 1(d) 
TABLe 1

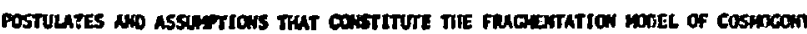

1. The coupresston thas eakes place in the pre-exploston collapse lests to:

2. Fragechtetion oceurs ot on serly stage of tha exponstion and results la

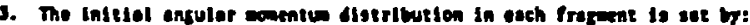

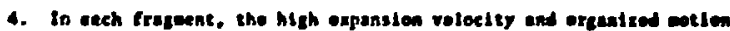

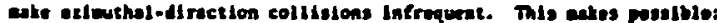

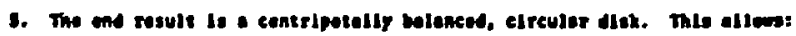

(a) aterial of uniforn denstty that exhibita soes degret of vistosity. axt

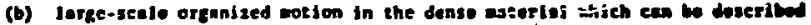
Jocally by e Itinesr relocity profils.

(a) frezents thet separate troe each other as expanslon prozrssues, and (b) eravitational binding of the partlcles elthin each frtgent volues.

(o) the tangentiol velociey pattem dorived fros 1(b). and

(b) the shope and erlentation of the fraguent volues.

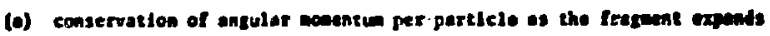
and subsenutatly cellepses snte a Alsh, and, thorefore,

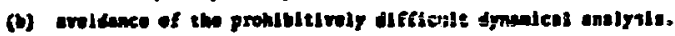

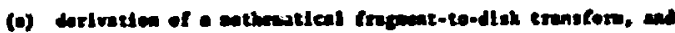

(b) cotententien of the milat ass distribution in tho disk.

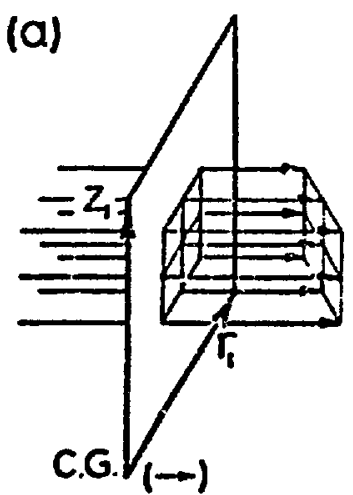

(b)

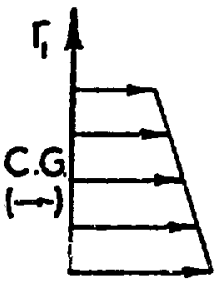

(c)

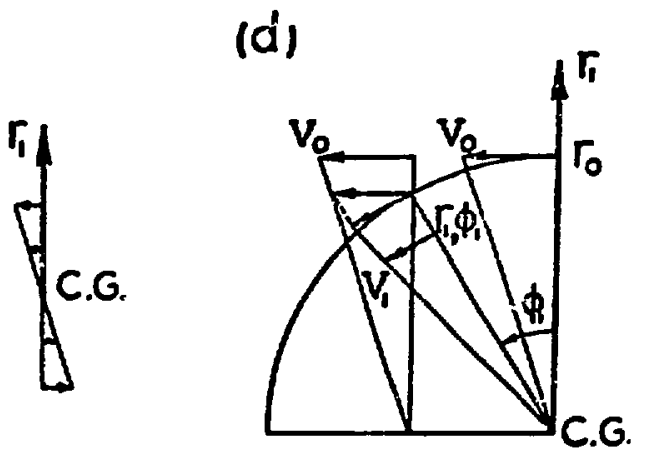

Fig. 1. Steps in the derivation of the tangential velocjty pattern within a fragment. (a) Orientazion of the $z_{1}$ and $r_{1}$ axes of the cylindrical coordinate system with respect tu the $1 \mathrm{arge-scale}$ mass flow: the origin is at the fragnent center of gravity (C.G.). (b) The linear velocity profile of the bulk flow. (c) The velccity profile in the $r_{1}, p_{1}$ plane, with respect to the fragnent center of gravity. (d) The tangential velocity, $v_{1}$, determined for a point $\left(r_{1}, ?_{1}\right)$.

shows that the tangertial velocity pattern is given by

$$
v_{1}\left(r_{1}, \phi_{1}\right)=v_{0} \frac{r_{1}}{r_{0}} \cos ^{2} \phi_{1} .
$$

The conservation of angular momentim per particle in the transition from frazment to disk is symbolized by

$$
r_{1} v_{1}\left(r_{1}, \phi_{1}\right)-r_{2} v_{2}\left(r_{2}\right)
$$

where $r_{2}$ describes radial position within the circular disk and $v_{2}$ is the orbital velocity:

$$
v_{2}\left(r_{2}\right)=\sqrt{\frac{G M_{c}}{r_{2}}} .
$$

A large central mass, $M_{C}$, is presumed to be present within the disk; $G$ is the gravitational constant. 
Combining Eqs. (2) through (4) shows that a particle initially located at position $\left(r_{1}, \phi_{1}\right)$ in the fragment eventually orbits in the disk at a radius $r_{2}$ given by the mapping transform:

$r_{1} \cos \phi_{1}= \pm\left(\frac{r_{0}^{2} G M_{c} r_{2}}{v_{0}^{2}}\right)^{1 / 4} \equiv \pm D\left(r_{2}\right)$.

The defined length, $D$, is fixed for a particular $r_{2}$, so that, as seen from Eq. (5), the locations of the two plares of isoangular momentum are specified in the fragment coordinate system, $r_{1}, \phi_{1}, z_{1}$. According to the model, any material located within the fragment in either of these two planes is destined for orbit at $r_{2}$ within the disk. Figure 2 (a) depicts a fragment and shows the two planes of the transform. Figure 2 (b) shows the final configuration of the model on the galactic scale: a flat circular disk rotating in centripetal balance about a large central mass.

It is convenient to normalize the transform so that particles of maximum angular momentum initially located on the periphery of the fragment at $\left(r_{1 m}, \phi_{1 m}, z_{1 m}\right)$ will orbit at the edge of the disk at the maximum radius $\mathbf{r}_{2 m}$ :

$$
\frac{r_{2}}{r_{2 m}}=\left(\frac{r_{1} \cos \phi_{1}}{r_{1 m} \cos \phi_{1 m}}\right)^{4}=\left(\frac{D}{D_{m}}\right)^{4} \text {. }
$$

The length $D_{m}$ specifies the location of the plane farthest out from the center of mass, just touching the surface of the fragment boundary at the point of maximum angular momentum, as in Fig. 2(a).

In the disk, the mass $M\left(r_{2}\right)$ within the radius $r_{2}$ is given by the integration

$$
M\left(r_{2}\right)=\rho \int d V=2 \rho \int_{0}^{D\left(r_{2}\right)} A(D) d D,
$$

where $\rho$ is the mass density (uniform) within the fragment and where the integration

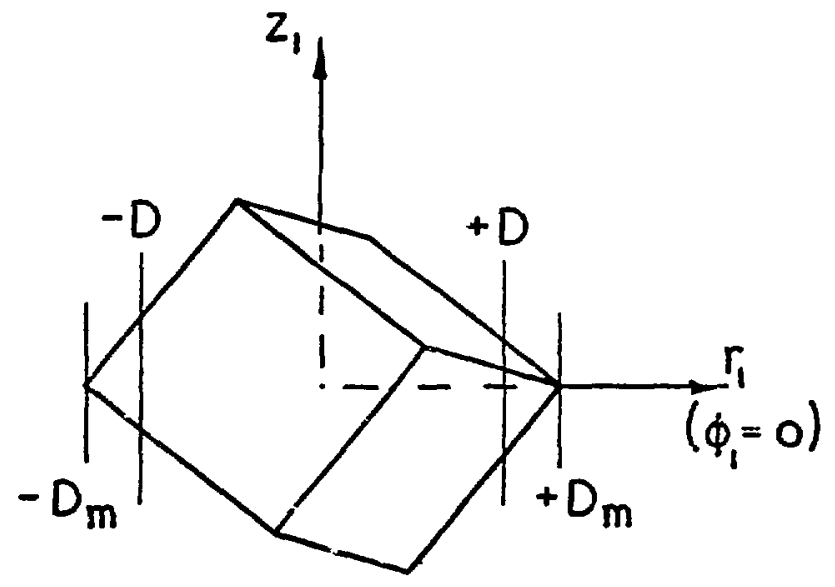

(a)

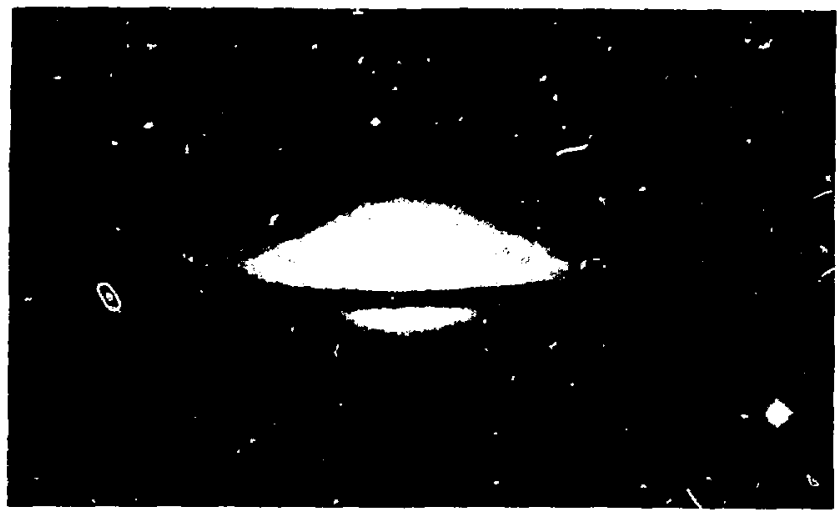

(b)

Fig. 2. The initial and final stages of galactic formation according to the fragmentation model. A fragment that packs to fill all space at the time of separation is illustrated by the cube in (a). The planes $\pm D$ of the mapping transform are shown edge-on. The final product of the model is a system like that shown in (b), a flat circular disk rotating in ceitripetal balance about a large central mass. (Photograph of NGC 4594 from the Hale observatories.) 
is performed over that region of the fragment volume contained between the two planes of the mapiing transform. The area of these isoangular momentum planes within the iragient is symbolized by $A(D)$.

The radial mass distribution, $m\left(r_{2}\right)$, in the disk is given by

$$
m\left(r_{2}\right)=\frac{\partial M\left(r_{2}\right)}{\partial r_{2}}=2 \rho A\left[D\left(r_{2}\right)\right] \frac{\partial D\left(r_{2}\right)}{\partial r_{2}}
$$

The surface mass distribution, $\sigma\left(r_{2}\right)$, is given by

$$
\sigma\left(r_{2}\right)=\frac{m\left(r_{2}\right)}{2 \pi r_{2}}
$$

IV. GALACTIC FORMATION ACCORDING TO THE MOPEL

It is postulated that at some time after the big bang, when matter and radiation have decoupled, the compressed matter of the universe separates into individual fragnent volumes. At the moment of breakup, the fragments must pack to fill all space and therefore, of necessity, must exhibit projections to make close packing possible. It is envisioned that, in genera1, the fragments will resemble a multitude of soap bubbles packed closely together. After separation, the material within each fragment volume is accelerated toward the fragment center of mass while the space itself continues to expand.

For analysis, a convenient choice for the fragment shape is a cube, because it is one of the shapes that packs to fill all space. Although the fragment's orientation with respect to the bulk flow would be random in general, Fig. 2 (a) shows a cubical fragmunt whose opposite corners chanced to be in one of the two locations of maximum angular momentum, at $\pm D_{m}$ where $\phi_{1}=0, \pi$. With any such cornerlike projections thus oriented, the area on the planes of isoangular momentum within the fragment is given by

$$
A(D) \propto\left[D_{m}-D\left(r_{2}\right)\right]^{2}
$$

Normalized to the total fragment mass, $\mathrm{M}_{\mathrm{T}}$, the corresponding mass distributions in the disk 5 are given by

$$
\frac{M\left(r_{2}\right)}{M_{T}}=1-\frac{9}{8}\left[1-\left(\frac{r_{2}}{r_{2 m}}\right)^{1 / 4}\right]^{3}
$$

and

$$
\frac{\sigma\left(r_{2}\right)}{\mathrm{M}_{\mathrm{T}} / \pi \mathrm{r}_{2 \mathrm{~m}}^{2}}=\frac{27}{\delta 4}\left(\frac{\mathrm{r}_{2}}{\mathrm{r}_{2 \mathrm{~m}}}\right)^{7 / 4}\left[1-\left(\frac{\mathrm{r}_{2}}{\mathrm{r}_{2 \mathrm{~m}}}\right)^{1 / 4}\right]^{2} .
$$

If the cubical fragment had been oriented so that two opposite edges 1 ay in the $p l$ anes of maximum angular momentum at $\pm D_{m}$, then the exponent in Eq. (10) would be unity and the mass distributions would change accordingly. If this exponent is symbolized by $v$, for a fragment oriented with faces lying in $\pm D_{m}, v=0$. Table II (from Ref. 5) 1 ists the mass distributions for these cases. Also shown in Table II, is the mass fraction located at the center within $0.1 \mathrm{r}_{2 \mathrm{~m}}$. This fraction shows that the cases $v=I$ and 2 have enough mass near the center to support the assumption of a large central mass underlying Eq. (4). The distributions for $v=0$, however, are seen to have a large fraction of the mass out in the disk. Such a distribution is characteristic of spiral galaxies, but, as a large central mass is not present, the condition implicit in Eq. (4) is not satis fied. Appendix A contains an investigation 
MODEL. PREDICTIONS FOR VARIOUS ORIENTATIONS OF CUBICAL, FRAGMENT FEATURES

Characteristics

$v$

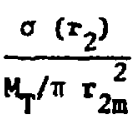

$\frac{n\left(r_{2}\right)}{M_{T} / r_{2 m}}$

$$
\frac{27}{32}\left(\frac{r_{2}}{r_{2 m}}\right)^{-3 / 4}\left[1-\left(\frac{r_{2}}{r_{2 m}}\right)^{1 / 4}\right]^{2}
$$$$
1-\frac{9}{8}\left[1-\left(\frac{r_{2}}{r_{2 m}}\right)^{1 / 4}\right]^{3}
$$

$\sim 91 \%$

9

$M(0.1) / M_{T}$

$\frac{27}{64}\left(\frac{r_{2}}{r_{2 m}}\right)^{-7 / 4}\left[1-\left(\frac{r_{2}}{r_{2 m}}\right)^{1 / 4}\right]^{2}$

$M\left(r_{2}\right) / M_{T}$

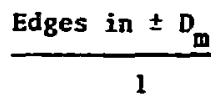

Faces in $\pm D_{m}$

0

$\frac{1}{4}\left(\frac{r_{2}}{r_{2 m}}\right)^{-7 / 4}\left[1-\left(\frac{r_{2}}{r_{2 m}}\right)^{1 / 4}\right]$

$\frac{1}{8}\left(\frac{r_{2}}{r_{2 m}}\right)^{-7 / 4}$

$1-\left[1-\left(\frac{r_{2}}{r_{2 m}}\right)^{1 / 4}\right]^{2}$

$\left(\frac{r_{2}}{r_{2 m}}\right)^{1 / 4}$

$\leq 81 \%$

$<56 \%$

$r_{2 m} /\left(\frac{v_{0}}{r_{0}}\right)^{2} \frac{a^{4}}{G r_{T}}$

$\frac{1}{2}\left(\frac{r_{2}}{r_{2 m}}\right)^{-3 / 4}\left[1-\left(\frac{r_{2}}{r_{2 m}}\right)^{1 / 4}\right]$

$\frac{1}{4}\left(\frac{r_{2}}{r_{2 \pi}}\right)^{-3 / 4}$

Tentative Designation:

Sphericals

Ellipticals

Spirals

a Distributions valid for $\frac{r_{2}}{r_{2 m}}>\frac{1}{81}$.

of the mass distributions near the disk center.

The disk radius may be derived from the model. In terms of fundamental variables it is:

$$
r_{2 m}=\left(\frac{v_{0}}{r_{0}}\right)^{2} \frac{a^{4}}{G M_{T}}(v+1)^{2}
$$

This indicates that for the same velocity gradient, mass, and cube size, spherical galaxies will be about nine times as large as normal spirals (see Table II).
We now consider the model's predicted mass distributions for elliptical galaxies. The mass distributions for the cases $v=1$ and 2 bear a strong resemblance to de Vaucouleurs' ellipsoidal 1 aw, ${ }^{6}$ an empirical description which states that the logarithm of the surface brightness varies 1 inearly with the radius to the one-quarter power. The one-quarter power variation in the model predictions arises directly from the mapping transform, Eq. (5) or (6). Curves of the surface mass distributions predicted by the model are shown in Fig. 3. These may be compared with the surface luminosity of the elliptical galaxy shown in Fig. 4. 


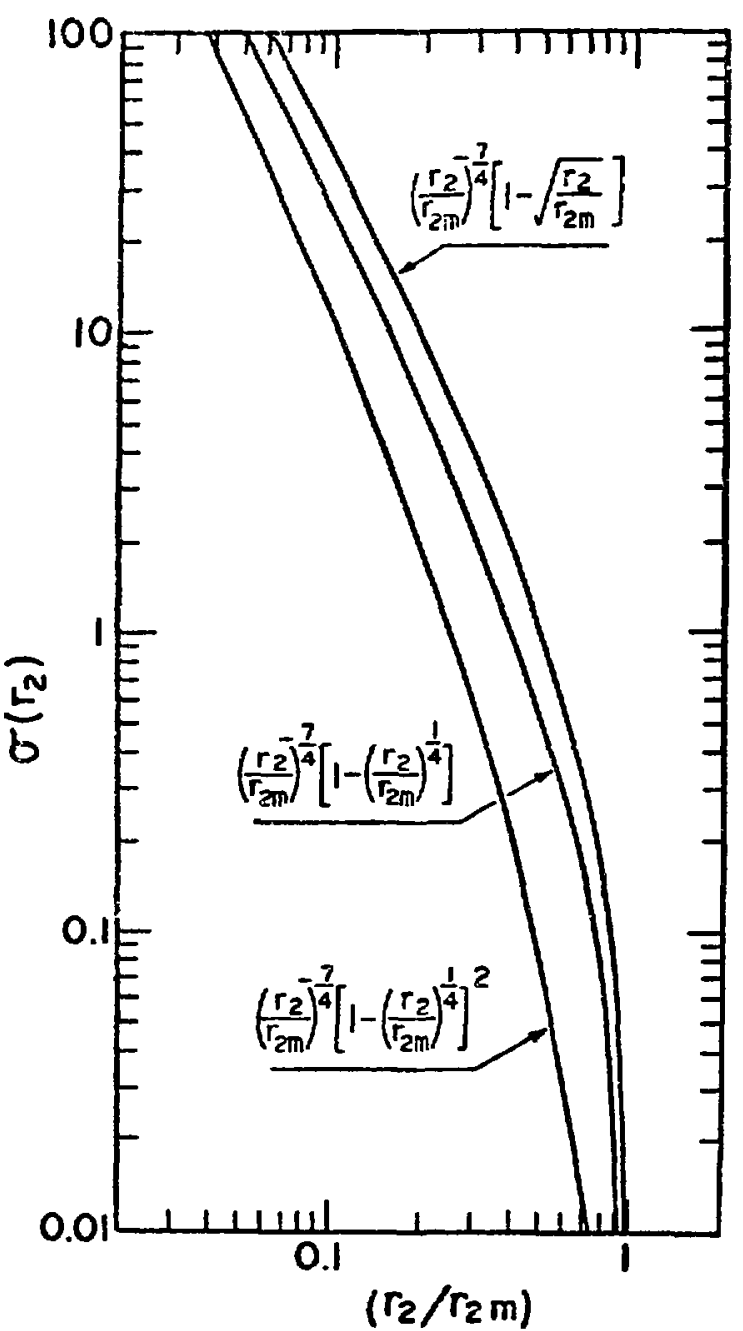

Fig. 3. Functions derived from the model to describe the distributions of surface mass density in elliptical galaxies. (Lower curvis: cubical fragment; upper curve: spherical fiagment, which does not closepack.)

The curve shapes are quite similar, and, in particular, the $v=1$ model curve segment $0.1<\mathrm{r}_{2} / \mathrm{r}_{2 \mathrm{~m}}<0.7$ fits the 1 ight intensity curve of Fig. 4 very well from $0<10 g$ I $<2$.

Note that, although a specific fragment shape (the cube) has been used, the

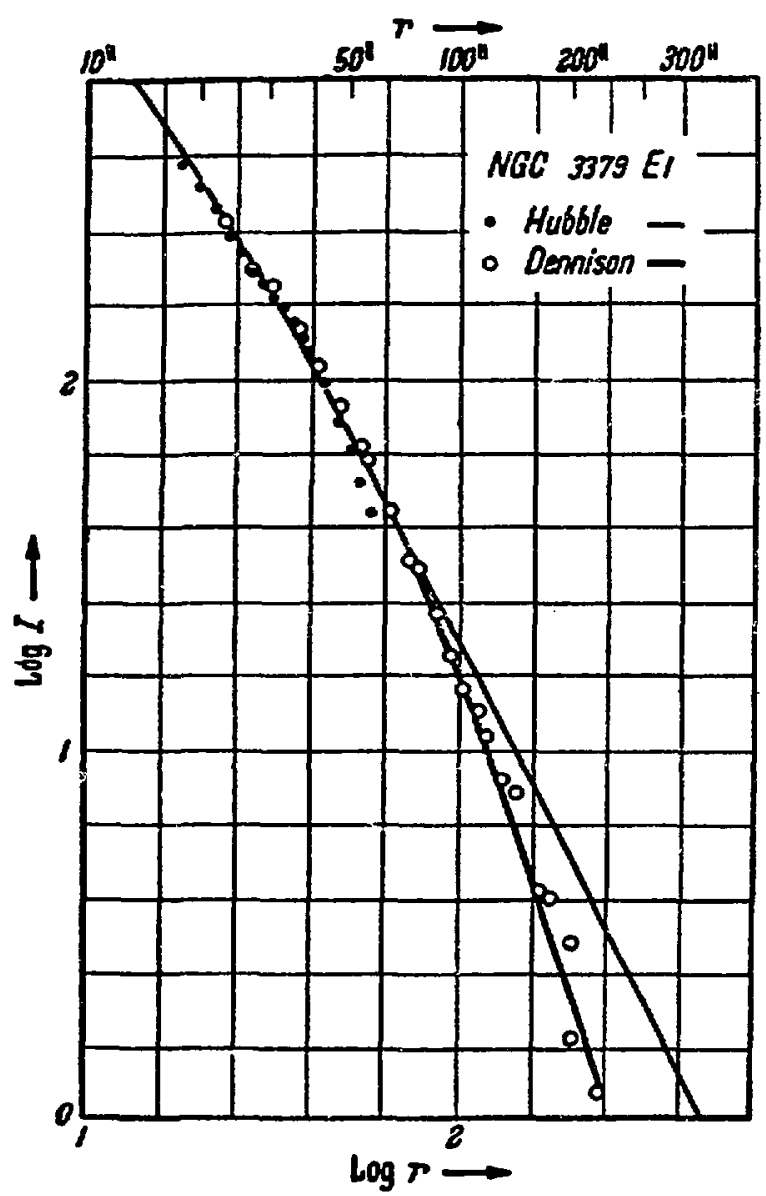

Fig. 4. Luminosity distribution in an e1liptical galaxy. (From de vaucoul eurs. ${ }^{8}$ )

fourth power of the mapping transform ensures that what dominates the mass distributions is the outermost features of the fragment volume that chance to be oriented near the two planes at $\pm D_{m}$. These are the two places in space where the material of maximum angular moment um is located. Thus, if another fragment shape were to be examined, any vertex or edge lying near the planes $\pm D_{m}$ would be mapped into the disk by the fourth power, and the resulting distributions would not differ appreciably from those of the cube. 
Al though the present formulation of the model precludes analysis of dynamic behavior in the process of galaxy formation, one can infer from the fact that the proposed initial pattern of angular momentum is two-sided, that intermediate configurations would probably display two major spiral arms. If the initial conditions are such that a relatively large amount of fragment mass lies near the planes $\pm D_{m}$ (e.g., $\nu=0$ ), then the two spiral arms in the resultant galaxy would be a dominant feature in the more massive and extended disk.

Table III is a comparison of the model predictions and observations on the galactic scale.
V. SOLAR SYSTEM FORMATION ACCORDING TO THE MODEL

Application of the fragmentation model on the solar system scale involves the postulated breakup of an ejected supernova shell into massive fragments. Each fragment expands and subsequently collapses to a disk to form a complete solar system. The concept is depicted in Fig. 5 .

The supernova is felt to be a good candidate for the event, which, through nucleosynthesis, may have produced the isotopic inventory that constitutes terrestrial matter. The supernova is a common cosmic event, and the more massive ones required

TABLE III

COMPARISON OF MODEL PREDICTIONS AND OBSERVATIONS ON THE GALACTIC SCALE

\section{Mode1 Predictions}

A. Extended spirals

1. The disk contains about $50 \%$ of the total galactic mass; the remaining $50 \%$ is in the nucleus.

2. The intrinsic two-sided symmetry of the model constitutes a natural mechanism for producing extended systems, with two major spiral arms.

B. Ellipticals

1. The functional dependency of the predicted surface mass densities is quite similar to the ellipsoidal 1 aw. ${ }^{a}$

2. The model produces curves of the surface mass density distributions.

3. Spherical galaxies (or giant ellipticals) should be about nine times as large as normal spirals.

\section{Observations}

1. The proportions are uncertain, but perhaps equally divided between galactic disk and nucleus.

2. The presence of two major arms is a common feature of spiral galaxies.

1. The surface Iuminosity profiles follow de Vaucouleurs' ellipsoidal 1 aw.

2. The observed surface luminosity profiles are well fit by the model predictions.

3. Giant ellipticals are approximately a factor of ten larger than normal spirals.

\footnotetext{
${ }^{a}$ Reference 6 .
} 


$$
\frac{r_{2} m\left(r_{2}\right)}{M_{T}}=1 / 4\left(\frac{1+(b / c) \tan \theta}{(b / c) \tan \theta}\right)\left(\frac{r_{2}}{r_{2 m}}\right)^{1 / 4} 1 / \pi\left[\cos ^{-1} \frac{r_{+}}{c}-\frac{r_{+}}{c} \sqrt{1-\left(\frac{r_{+}}{c}\right)^{2}}\right]
$$

where

$$
\frac{r_{+}}{c} \equiv(1+(b / c) \tan \theta)\left(\frac{r_{2}}{r_{2 m}}\right)^{1 / 4}-(b / c) \tan \theta
$$

for

$$
\frac{r_{2}}{r_{2 m}}>\left(\frac{1-(b / c) \tan \theta}{1+(b / c) \tan \theta}\right)^{4} \text { and } 0<(b / c) \tan \theta<\infty \text {. }
$$

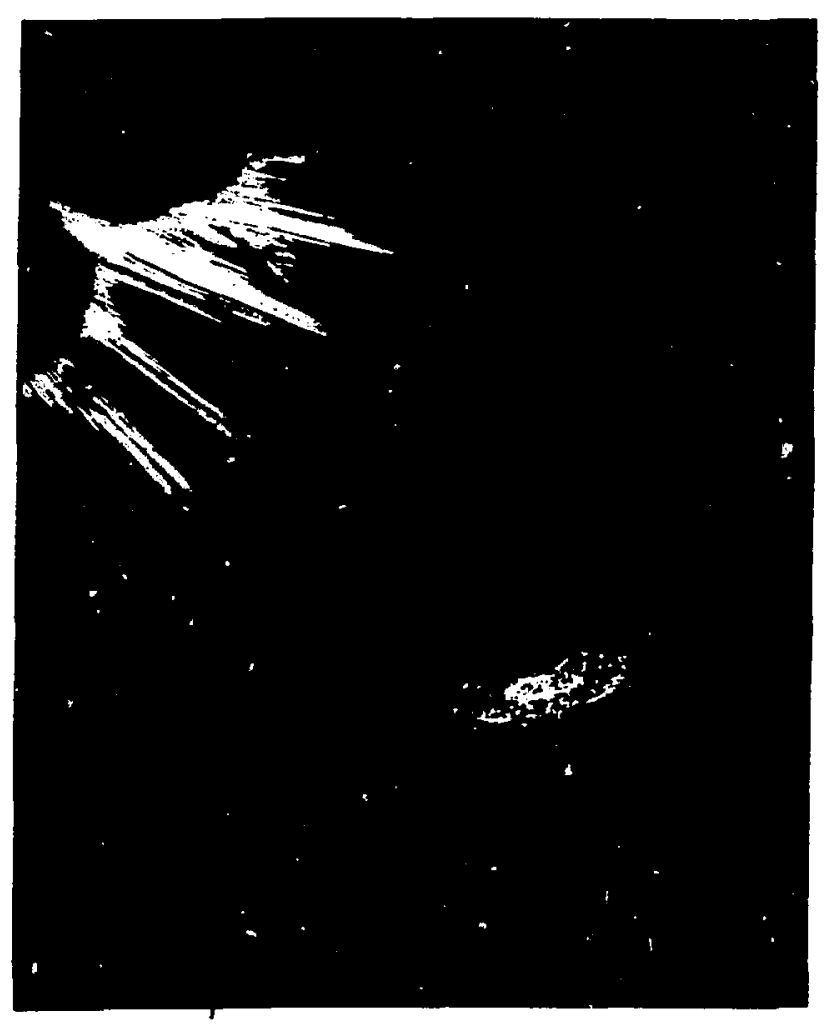

Fig. 5. An artist's conception of the initial and final stages of solar system formation according to the fragmentation model. The breakup of the expanding supernova shell into massive fragments is depicted at upper left. At right, rings of higher density can be seen within the planetary disk formed from one fragment.

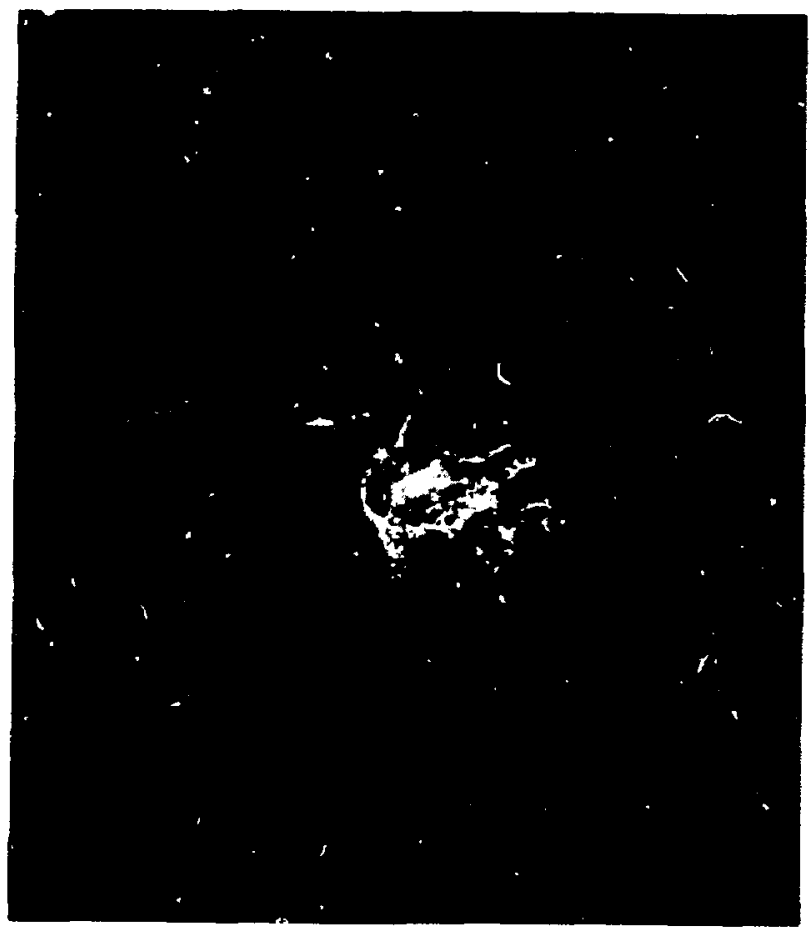

Fig. 6. The "Crab" nebula. This recent supernova appears to have ejected relatively Iittle mass, but it clearly displays structure. The event required by the model would have to eject a far more massive she11. (Photograph of NGC 1952 from the Hale Observatories.)

by this model may have been far more frequent earlier. The Crab nebula, a relatively recent supernova, is shown in Fig. 6 . Supposing that solar systems arise directly from supernovae does not raise the problem of having to presume that our solar system is a unique, or even an unlikely, phenomenon.

\section{For analysis, the typical supernova} shell fragment shape has been chosen to be a right circular cylinder. The flat faces are portions of the inner and outer spherical surfaces of the ejected supernova shell. 
The half-thickness of the shell is designated by $b$, and the cylinder radius by $c$ (see inset, Fig. 7). The cylindrical fragment is ejected outvard along the direction of the cylinder axis at, an angle 0 with respect to the rotational axis of the presupernova star. Application of the mapping transform of the model to this fraguent yields Eq. (14), the radial mass distribution $^{7}$ which deseribes the intersection of a plane with the lip of a tilted cylinder.

The variables $b, c$, and $\theta$ appear together only in the combination $(b / c) \tan \theta$, which parameterizes this solution. All the distributions in the range $0.5<(b / c)$ tan $\theta<1$ are virtuslly identical, and this mass distribution is the nost likely to be produced by the model. It is very encouraging that this most probable mass distribution is also that which best fies our large planess. (Comparison to Mercury, Yenus, Earth, and Nars is complicazed by the fact that something clearly differcnt. or even catastrophic, has accompanied the formation of these planets.) Figure 7 shows this distribution, and includes the outcr planets for comparison. Comparison is made possible by the fact that the plancts are

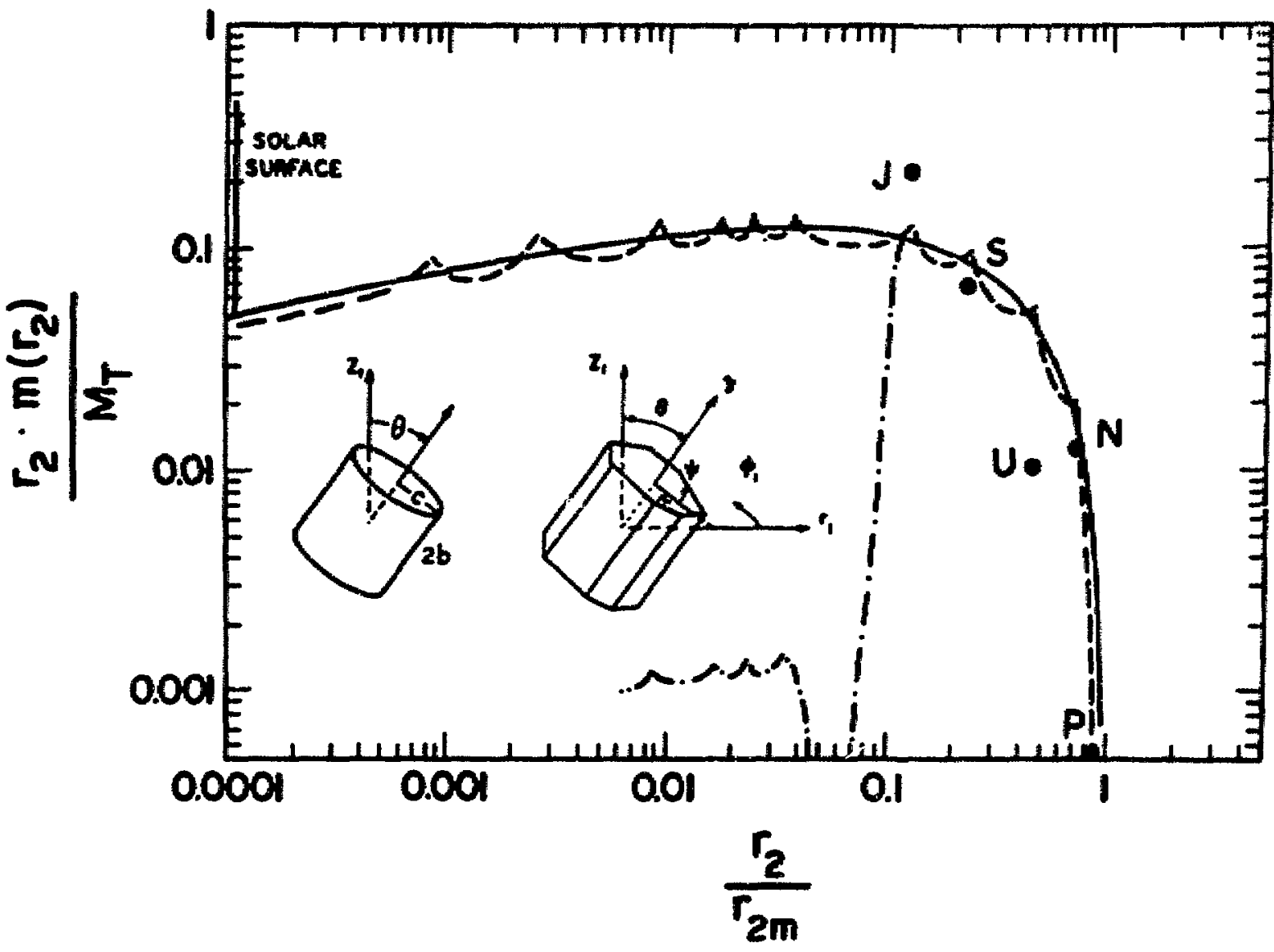

Fig. 7. The radial mass distribution (solid line) in the planetary disk yielded by any cylindrical fragment for which $0.5<(b / c)$ tan $\theta<1$ (see left inset). The large planets of our solar system are ploted for comparison. The dashed Iine is a sketch of the mass distribution that would result from a more realistic prism-ehaped fragnent (see right inset). The dashed-doted 1 ine is the type of mass distribution that could result from the presence of an opposing mass flow. 
very noarly crenly spaced in the logarieluic sense. Ploteing the plances' masses versus the logatith of the tadius therefore gives units of mass per logarithmic unit: just the dinension of the quantity thus chosen for displayifig the mass distribution, $r_{2} \mathrm{n}\left(x_{2}\right)$.

The mass distribution at the disk center is not sensitive to the fragment shape. Integration to find $M\left(r_{2}\right)$ ard $L\left(r_{2}\right)$. the integrated angular momentum, reveals that about one-third of the system mass lies within $\tau_{2} / r_{2 m}=0.001$, but only -2 : of the angular momentum does $50 .{ }^{7}$ (Allercury's orbie lies about ten tines further out than this.) One-third is a relatively large mass Eraction. athough it is by no means cqual to the overwhelming fraction found in our sun. It will be shown shorty that the model contains a mechanism capable of supplying much more mass to the sun from the central region of the disk \{c.g., out to Jupiter) without adding angular momentun.

To recurn to the question of the planetary mass distribution, it must be realizod that the cylindrical fragment shape is somewhat idealistic. The fragments must fit together and close-pack to account for the cntirc spherical shell surface (in the same sense that on the galactic scale, the fragments must pack to fill all space). A more realistic fragment shape is therefore a polygenal cylinder or prism. An example of polygonal shapes being produced in the dezonation of a high explosive is shown in Fig. 8 .

The mass distribution yielded by a prism-shaped fragment will be generally similar to that of a cylindrical fragment of the same proportions, but the vertices and flats will cause the curve to swing above and below the smooth distribution to produce a series of maxima and minima. Even if small, the maxima will predispcse the disk to condense into planets in this pattern.

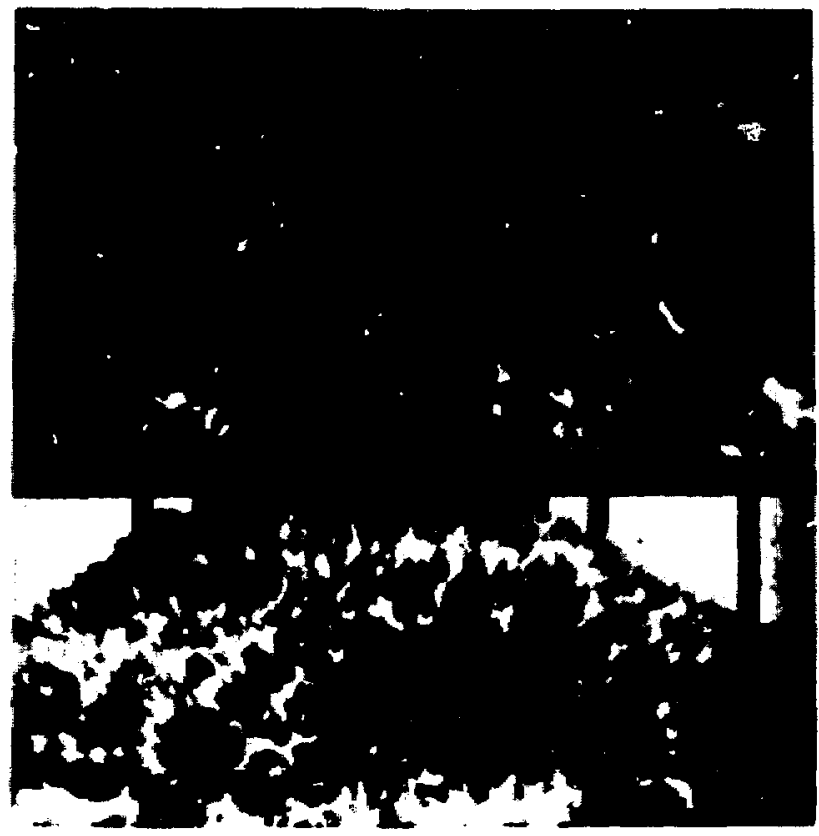

Fig. 8. A stop-motion plotograph of a lromogeneous metal shell driven outward by the detonation of a structureless ligh explosive within. incanciescent gasses have passed outward between the metal fragments and out line the underlying polygonal shapes. A mirror positioned above at $45^{\circ}$ affords $z$ view from the top. (The unusual phenomena at the top, caused by special conditions of this experiment, are not pertinent and should be disregarded.)

It is a difficult analytical task to map the prism-shaped fragment into a disk distribution, but a relatively easy one to determine the radii in the disk corresponding to the prism vertices. It is convenient to establish a new cylindrical coordinate system $(r, \psi, 2)$ as shown in the inset in Fig. 7, in which system the $j$ vertices are located at $n_{j}, \psi_{j}$ and $\pm b$. When these points are mapped into the disk with the transform, the follewing series results.

$r_{2}($ A.U. $)=0.418\left( \pm 1+\frac{\cos \psi_{j}}{\left(b / r_{j}\right) \tan \theta}\right)^{4}$, 
where the coefficient 0.478 has been chosen to provide a reasonable fit to our solat system. This expression produces a series that provides a natural fit for the orbits of our planets. Of course, to cescribe the spacing of planets within our particular solar system, one must choose a specific polygonal shape. If we choose a regular heptagon for which $\left(b / r_{j}\right) \tan \theta=0.466$, i.e. $(b / c) \tan \theta=0.5$, and set $\psi_{1}=(1 / 8)$ $\left(360^{\circ} / 7\right)=6.4^{\circ}$, the series produced is 0.2 , $0.5,0.8,1.1,4.1,10,19,30$, and 40 A.U. (The vertex of maximum angular momentum places the disk edge at about 46 A.U.; four others are at radii $<0.2$ A.U., making a total of 14 maxima, corresponding to the 14 vertices of the heptagonal prism.) Because of the fourth-power dependency in the mapping transform, slight shifts of the vertices away from the regular heptagon positions can produce an exact fit to our solar system. A sketch of the mass distribution with maxima and minima is included in Fig. 7 .

The model also predicts that the ostermost planet should have a tilted, eccentric orbit, as does pluto. Unlike all other vertices, the one representing the last planet has no fragment material on the opposite side of the midplane $(z=0)$ to mix with. This mass can therefore settle into an orbit at an angle to the midplane. Up to this point, analysis has been based on the hypothesis that, before fragmentation, the bulk flow in the supernova shell was plane parallel, as shown in Fig. 1. This would be the case if, for example, the dimensions of the fragment were small compared to the distance, $R_{0}$, of the fragment from the rotational axis of the presupernova star. Actually, the preexplosion rotation of the shell adds a term to the expression for the pattern of tangential velocity with respect to the fragment center of mass. Equation (2) becomes $v_{1}\left(r_{1}, \phi_{1}\right)=v_{0} \frac{r_{1}}{r_{0}} \cos ^{2} \phi_{1}-v\left(R_{0}\right) \frac{r_{1}}{R_{0}}$.

The additional term is just the scaled-down tangential velocity, $V(R)$, of the shell itself with respect to the axis of rotation of the presupernova star.

Al though the mapping transform has exactly the same form wheiher $v_{0} / r_{0}$ is positive or negative, is profile has been chosen which decreaseri with $R$ 'snd, hence, the minus sign in Eq. (16). The effect is to create a second rass fiow in the separated fragment, rotating in the opposite sense to that resulting from the velocity profile of the bulk flow. While the fragment is expanding into a gaseous cloud, particle collisions will be infrequent. However, as the cloud collapses to a disk, the two opposing flows will clash and produce a great deal of low-angular-momentum material that will be delivered to the sun as it assembles.

Under a wide range of conditions, the opposing flow will affect only the central region of the disk, and even there it will be dominated by the "forward" flow. The mass distribution in the outer disk will not be affected, but the inner disk may be altered, as sketched in Fig. 7. The small amount of material remaining in orbit in the central region will be enriched in heavy elements and will eventually form smal1, dense planets nearest the new sun. 8

The results discussed above are summarized in Table IV, along with some more speculative conclusions based on the presence of an opposing flow.

The process of solar system formation has not yet been observed, or, if it has, has not been recognized as such. The descriptive success of this model suggests that we might re-examine carefully both gassy stars receding from supernova sites and small (G-, K-, and M-type) stars with high space velocities, for evidence. 
TABLE IV

MODEL PREDICTIONS ON THE SOLAR SYSTEM SCALE

Plane-Parallel flow (from assumed linear velocity profile alone)

1. The planets should revolve about the sun in the same direction in circular, coplanar orbits.

2. The most probable mass distribution best fits the large planets (derived using a right circular cylindrical fragment).

3. A central mass containing only 1 to 24 of the angular momentum, but with a sizeable fraction of the system mass may be assembled.

4. The last planet should have a tilted, eccentric orbit, as does Pluto.

5. The vertices of a realistic, prism-like fragment produce a series of maxima in the radial mass distribution which provides a natural fit to the actual spacing of our planets.

$\overline{a_{\text {This }}}$ is a direct result of postulating that the end result is a disk.
Plane-Parallel Flow Plus Rotation of the Presupernova Star

1. Most of the hydrogen and helium is removed from the central region of the disk and delivered to the forming sun, adding little or no angular momentum, however, because the opposing flows have cancelled in this respect. Neither the mass distribution in the outer disk nor the locations of the maxima are affected.

2. The small fraction of material remaining in orbit in the central region of the disk is enriched in heavy elements and will form small, dense planets nearest the new sun.

3. Other, more conjectural, consequences of the reverse flow are:

a. A local manifestation of the reverse flow may result in a slow, retrograde planetary rotation, like that of Venus.

b. If the two flows are approximately equal in a region, all mass may be removed, as in the gap between Mars and Jupiter.

c. A maximum in the reverse flow may result in a distribution that would lead to Mars' small mass and the formation of Earth's moon.

d. Manifestations of the reverse flow in the minima in the outer region of the disk could lead to the formation of out $r$ moons in retrograde orbits such as those of Jupiter, Saturn, and Neptune.

e. The outer edge of the reverse flow could be responsible for the anomalous rotation of Uranus and its moons. 
VI.

SLIMAARY AND CONCLUSION

A new cosmogonical model has been formulated and successfully applied to both galactic and solar system formation. The postulates of this model and the results of its application on these widely differing scales are reviewed here. Considerable strength accrues to the model because of this broad applicability, which allows it to be tested against both the available information on elliptical galaxies and the detailed information about our solar system.

The central concept of the model is fraguentation, a postulated event that defines the angular momentun distribution within each fragment at the moment of breakup. The final distribution in a centripetally balanced disk is inferred directly from this initial distribution without treatment of the dynamics of formation. On both the galactic scale and the solar system scale, after fragmentation the model depicts the fragment material as being accelerated toward the fragment center of mass while expansion of the space continues. The model does not address the problem of identifying the actual mechanism, or mechanisms, by which each element of mass within a fragment is delivered to orbit in the disk.

The degree of success thus far

achieved in application of the model suggests that further efforts are warranted. Such efforts will inevitably involve computer calculations, as the project has reached the stage where analysis is cumbersome. With the kind assistance of F. Hohl of Langley Research Center, a program is presently being developed to simulate galactic formation dynamics following initial conditions proposed in the model. The velocity distribution described by the basic Eq. (i) will be superimposed on the material contained in a cubical fragment in various specified orientations. In addition to examination of intermediate configurations assumed by the mass during the fragment-to-disk transition, the program will allow comparison of the disk formed under dynanic conditions to that calculated as in this report using the assumption of the presence of a large central mass. The mass distribution for the spiral case may be obtained by this method, whereas it appears that it cannot by the present method. The conditions for dynamic stability in the disk will be investigated.

Regarding solar systems, where the large central mass assumption is not in question, a computer program is planned that will allow the mapping of realistically shaped supernova shell fragments to determine the relatively complex mass distributions in the disk. The model may be reapplied to the successive maxima of this distribution to predict the characteristics of planet-moon systems.

Another promising extension of the model is a statistical study of the probability of occurence of various morphological types of galaxies. This probability could be investigated by studying the spectrum of mass distributions yielded by a cubical fragment oriented randomly with respect to the prefragmentation bulk flow. Concurrence with reality in this study would suggest that a statistical study of the mass distributions resulting from a random array of supernova shell fragments could yield a reasonable prediction of the probable morphology of solar systems as yet unobserved. 
PREDICTED MASS DISTRIBUTIONS IN THE GALACTIC DISK CENTER

Brandt and Belton ${ }^{9}$ have introduced the following generalized rotational velocity relationship for nalyzing the observed rotational curve of galaxies.

$$
v(r)=\frac{3^{3 / 2 n} v_{\max } \frac{r}{r_{\max }}}{\left[1+2\left(\frac{r}{r_{\max }}\right)^{n}\right]^{3 / 2 n}},
$$

where $v(r)$ is the circular velocity at a distance $r$ from the rotation axis, $n$ is a numerical index, $v_{\max }$ is the maximum rotational velocity of the galaxy, and $r_{\max }$ is the corresponding distance from the axis. For $s$ mall values of $r / r_{\max }$, we find

$$
v(r)=3^{2 / 3 n} v_{\max } \frac{r}{r_{\max }} .
$$

For large values of $r / r_{\text {max }}$,

$$
v(r)=\left(\frac{3}{2}\right)^{3 / 2 n} v_{\max }\left(\frac{r}{r_{\max }}\right)^{-1 / 2},(A-3)
$$

where the functional dependence on $r$ is just as it would be if a large central mass were present.

From the text, we may recall that the velocity profile in a fragment with respect to the fragment center of mass is

$$
v_{1}\left(r_{1}, \phi_{1}\right)=v_{0} \frac{r_{1}}{r_{0}} \cos ^{2} \phi_{1}
$$

Conservation of angular momentum per particle in the transition from fragment to disk is symbolized by

$$
r_{1} v_{1}\left(r_{1}, \phi_{1}\right)=r_{2} v_{2}\left(r_{2}\right)
$$

We use the definition of the length, $D$, as in the text:

$$
D\left(r_{2}\right) \equiv r_{1} \cos \phi_{I}
$$

which represents the planes of isoangular momentum from which come particles in the fragment destined for circular orbit in the disk at $r_{2}$. By combining Eqs. (A-4) through $(A-6)$, we find that

$\pm D\left(r_{2}\right)=r_{1} \cos \phi_{1}=\sqrt{\frac{r_{0}}{v_{0}} r_{2} v_{2}\left(r_{2}\right)}$,

so that if we know the dependence of $v_{2}$ on $r_{2}$ we can derive a fragment-to-disk mapping trans form.

The text summarizes the results of using the large central mass assumption, symbolized by Eq. (A-3), where the mapping transform is

$$
\frac{r_{2}}{r_{2 m}}=\left(\frac{D}{D_{m}}\right)^{4}
$$

The resulting mass distributions, valid in the disk but not near the center, have been explored in the text.

We may also investigate the model prediction of mass distribution near the disk center by substituting Eq. (A-2) into (A-7). The resulting mapping transform is

$$
\frac{r_{2}}{r_{2 m}}=\left(\frac{D}{D_{m}}\right)
$$


and we can derive the central mass distributions using

$$
M\left(r_{2}\right)=\rho \int_{-D}^{+D} A(D) d D
$$

where $M\left(r_{2}\right)$ is the cumulative mass in the disk center out to $r_{2}, p$ is the density (uniform), and $A(D)$ is the area on the isoargular momentum $p l$ anes inside the fragment at $\pm D$.

In general, if $v_{2} \propto r_{2}$ at the disk center, using

$$
A(D) \propto\left[D_{m}-D\left(r_{2}\right)\right]^{\nu}
$$

we find that

$$
\begin{aligned}
& \frac{M\left(r_{2}\right)}{M_{T}} \propto 1-\left[1-\left(\frac{r_{2}}{r_{2 m}}\right)\right]^{v+1}, \\
& \frac{m\left(r_{2}\right)}{M_{T} / r_{2 m}} \propto(v+1)\left[1-\left(\frac{r_{2}}{r_{2 m}}\right)\right]^{v},
\end{aligned}
$$

and

$$
\frac{\sigma\left(r_{2}\right)}{M_{T} / \pi r_{2 m}^{2}} \propto(v+1)\left(\frac{r_{2}}{r_{2 m}}\right)^{-1}\left[1-\left(\frac{r_{2}}{r_{2 m}}\right)\right]^{\nu}
$$

The most critical case is that for the spiral galaxies where such a large fraction of the mass is out in the disk. For this case, where $v=0, A(D)$ is a constant and

$$
M\left(r_{2}\right) \propto \frac{r_{2}}{r_{2 m}}
$$

and

$$
\sigma\left(r_{2}\right) \propto\left(\frac{r_{2}}{r_{2 m}}\right)^{-1}
$$

'he expression for the surface density in the center, given by Eq. $(A-17)$, may be compared to that derived in the text and applicable out in the disk:

$$
\sigma\left(r_{2}\right) \propto\left(\frac{r_{2}}{r_{2 m}}\right)^{-7 / 4}
$$

Proceeding from the disk center toward the outer edge, there would be a smooth transition from the description given by Eq. $(A-17)$ to that given by Eq. $(A-18)$.

\section{REFERENCES}

1. M. M. Woolfson, "The Evolution of the Solar System," Rep. Progr. Phys. 32 , 135 (1969).

2. J. H. Oort, "The Formation of Galaxies and the Origin of the High-Velority Hydrogen," Astron. Astrophys i, 381 (1970).

3. L. Mestel, "On the Galactic Law of Rotation," Mon. Notices Roy. Astron. Soc. 126, 553 (1963).

4. W. K. Brown, "A Solar System Formation Model Based on Supernova Shell Fragmentation," Icarus 15, 120 (1971).

5. W. K. Brown, "A Galactic Formation Model Based on Post-Big-Bang Fragmentation," Astrophys. Space Sci. 15, 293 (1972).

6. G. de Vaucouleurs, "General Physical Properties of External Galaxies" in Handbuch der Physik, Vol. LIII, S. FIugge, Ed. (Springer-Verlag, Beriin, Götingen, Heidelberg, 1959), p. 311 . 
7. W. K. Brown, "A Model for Formation of Solar Systems from Massive Supernova Fragments," Los Alamos Scientific Laboratory report $\mathrm{LA}-4343$ (1970).

8. W. K. Brown, "Particle Collisions in the Opposing Mass Flow Hypothesis: A Facet of the Supernova Fragmentation
Model of Solar System Formation," Los Alamos Scientific Laboratory report LA-4343 Supplement (1971).

9. J. C. Brandt and M. J. S. Belton, "On the Distribution of Mass in Galaxies. III. Surface Densities," Astrophys. J. 136,352 (1962). 